\title{
Evaluation of an Intervention to Reduce Tear Gas Exposures and Associated Acute Respiratory Illnesses in a US Army Basic Combat Training Cohort
}

\author{
Joseph J. Hout ${ }^{*}, 1$, Duvel W. White ${ }^{1}$, Michael Stevens ${ }^{1}$, Alex Stubner ${ }^{1}$, Anthony Arino ${ }^{1}$ and Joseph Knapik ${ }^{2}$ \\ ${ }^{1}$ Uniformed Services University of the Health Sciences, 4301 Jones Bridge Rd, Bethesda, MD 20814, USA \\ ${ }^{2}$ United States Army Institute of Public Health, 5158 Blackhawk Rd, Aberdeen Proving Ground, MD 21005, USA
}

\begin{abstract}
Exposing unmasked US Army recruits to elevated levels of o-chlorobenzylidene malononitrile (CS tear gas) during Mask Confidence Training (MCT) increases the risk of Acute Respiratory Illness (ARI) diagnosis in the period following CS exposure when compared to the period before exposure. All Army Activities Message (ALARACT) 051/2013 was implemented in March 2013 to reduce CS exposure concentrations during MCT and associated ARI rates. This observational, prospective cohort studied CS exposures and associated ARI health outcomes after implementation of ALARACT 051/2013 in 5298 recruits attending US Army Basic Combat Training (BCT). These data indicate a 10-fold reduction $(\mathrm{p}<0.001)$ in CS exposure concentrations; recruit exposures ranged from $0.26-2.78 \mathrm{mg} / \mathrm{m}^{3}\left(\bar{x}=1.04 \mathrm{mg} / \mathrm{m}^{3}\right)$ and chamber operator exposures from $0.05-2.22 \mathrm{mg} / \mathrm{m}^{3}\left(\bar{x}=1.05 \mathrm{mg} / \mathrm{m}^{3}\right)$. The overall risk of ARI diagnosis following CS exposure also decreased when compared to period before exposure $(\mathrm{RR}=1.79,95 \% \mathrm{CI}=1.29,2.47)$ resulting in $26.85 \%$ $(95 \% \mathrm{CI}=-0.17,0.54)$ intervention effectiveness. Post-chamber ARI rates were dependent upon CS exposure concentration $(\mathrm{p}=0.02)$, and pre/post-chamber ARI rate ratios were significantly elevated at all concentration categories higher than the Threshold Limit Value Ceiling (TLV-C) $\left(0.39 \mathrm{mg} / \mathrm{m}^{3}\right)$. Results support previous research suggesting risk of ARI diagnosis after CS exposure is positively associated with CS concentration.
\end{abstract}

Keywords: CS, tear gas, riot control, o-chlorobenzylidene malononitrile, mask confidence.

\section{INTRODUCTION}

All recruits entering the United States (US) Army are exposed to the riot control agent o-chlorobenzylidene malononitrile (CS) during mandatory Mask Confidence Training (MCT) in Basic Combat Training (BCT) and throughout their careers during annual Chemical, Biological, Radiological and Nuclear (CBRN) refresher training [1,2]. MCT takes place in a relatively air-tight structure commonly referred to as the gas chamber, where a CS-rich environment is created and maintained by thermally dispersing CS on an improvised aerosol generator. Soldiers wearing military respirators equipped with filters designed to protect against airborne chemical warfare agents enter the chamber, perform exercises specified by instructors inside the chamber, and finally remove their protective masks before exiting the chamber [3, 4]. Soldiers feel almost immediate effects of CS upon mask removal (burning of the respiratory system, lacrimation, coughing, and sometimes vomiting) which demonstrates how a properly fitted protective mask shields the user from airborne chemical hazards [5, 6].

A recent study of 6723 recruits attending Army BCT at Fort Jackson, SC showed the standard Army method used to conduct the MCT resulted in exposure of $100 \%$ of the study

*Address correspondence to this author at the Department of Preventive Medicine and Biometrics Uniformed Services University of the Health Sciences 4301 Jones Bridge Road, Bethesda, MD 20814, USA; Tel: 410-733-5134/301319-6953; Fax: 301-295-0933; E-mail: joseph.hout@usuhs.edu population to CS concentrations exceeding the American Conference of Industrial Hygienists (ACGIH) Threshold Limit Value-Ceiling (TLV-C) $\left(0.39 \mathrm{mg} / \mathrm{m}^{3}\right)$. In addition, $98 \%$ of the study population also exceeded the limit $(2.00$ $\mathrm{mg} / \mathrm{m}^{3}$ ) deemed Immediately Dangerous to Life and Health (IDLH) by the National Institute for Occupational Safety and Health (NIOSH) [7-9]. Researchers discovered recruits were exposed on average to CS concentrations approximately five times higher than IDLH. Measured exposure levels ranged from $1.74-55.24 \mathrm{mg} / \mathrm{m}^{3}$ and recruits were exposed for periods as long as two minutes without respiratory protection. Investigators recommended several industrial hygiene controls to protect recruits including lowering CS concentrations, increasing personal protective equipment, and reducing unprotected recruit exposure time to 15 seconds [9].

In a concurrent research effort, recruits from the aforementioned study were observed to determine whether elevated CS exposure concentrations were associated with increased incidence of Acute Respiratory Illnesses (ARI) in Army BCT. Researchers found 2.44 times higher risk of recruits being diagnosed with ARI in the seven-day period following exposure to CS when compared to the seven-days preceding exposure. The observed increased ARI risk was independent of week of training or the type of building they lived in. Post-exposure ARI incidence was dependent on CS exposure concentration and pre/post-exposure risk ratios appeared to increase with concentration to a threshold; however a statistically significant dose-response relationship 
could not be established. Researchers concluded that lowering CS exposure concentration in the MCT could lower ARI rates and decrease lost training time and health care costs within this BCT population [10].

Preliminary results of both of these studies were presented to US Army medical and training officials through the Army Public Health Command (APHC), resulting in the publication of All Army Activities (ALARACT) message $051 / 2013$. ALARACT 051/2013 was implemented across the US Army in March 2013 as an intervention to address the elevated CS concentrations and ARI risks found at Fort Jackson. The ALARACT incorporated several recommendations made by researchers including reduction of CS used during MCT and maximum 15 second unprotected exposure times. In addition, it mandated semiannual industrial hygiene surveys and periodic wet cleaning of MCT chambers across the Army [11]. The present study was conducted after implementation of ALARACT $051 / 2013$ and was designed to evaluate the efficacy the intervention to lower CS exposure concentrations and MCT associated ARI risk.

\section{METHODS}

This observational, prospective cohort study quantified CS exposure concentrations as well as incidence and distribution of ARI before and after CS exposure to explore the association between CS exposure concentrations and ARI health outcomes in a gender integrated cohort of 5298 recruits completing mandatory MCT during US Army BCT at Fort Jackson, S.C. from 13 March to 30 April 2013. It further compared both CS exposure concentrations and ARI incidence results with those observed before implementation of ALARACT 051/2013 to evaluate intervention effectiveness. The study protocol was approved by the US Army Training and Doctrine Command (TRADOC) and the Uniformed Services University (USU) Office of Research, and was considered non-human subject research by the USU Institutional Review Board (IRB) [12].

Mask confidence training was scheduled by BCT companies (groups of approximately 200 recruits) through coordination with CBRN staff operating the Fort Jackson MCT. Typically, training was scheduled during the first three weeks of BCT at the rate of one company per day. Fort Jackson training officials provided investigators with company MCT dates, BCT start dates, and barracks types by unit identification code (UIC) prior to each scheduled MCT event.

On the day of the mask training, CBRN staff divided training units into four exposure groups of about 50 recruits each due to the limited size of the mask confidence chamber (described by Hout et al.); investigators did not influence exposure group size or composition [10]. Groups were then staged outside of the mask confidence chamber where they donned their protective masks and were inspected for functionality by CBRN staff prior to entering the chamber [9].

CBRN staff pre-heated a metal container to a mean temperature of $199^{\circ} \mathrm{C}$ on an electric hot plate (General Electric model\# 169214) positioned on a $1.10 \mathrm{~m}$ metal table in the center of the chamber. After approximately five minutes, the contents of three $650 \mathrm{mg}$ CS capsules were poured directly into the container causing immediate CS dispersion. CS was not agitated with a stirring rod as observed in previous studies and visibly aerosolized in less than three minutes. The first exposure group then entered the mask confidence chamber, lined up against the walls, performed various exercises specified by training staff, and finally removed their protective masks before exiting the chamber. CBRN staff then opened additional CS capsules and poured the contents in the metal container at the rate of one capsule for every 50 recruits exiting the chamber. The next group then entered and training continued in this manner until the entire BCT company completed chamber training.

Military trainers from each company provided rosters to document attendance, exposure group assignment (1-4), and mask confidence chamber completion. Recruits were included in the study population if they completed the mask confidence chamber with their assigned BCT company. Investigators were provided a count of recruits by unit identifier and exposure group upon successful completion of the mask confidence chamber at the end of each training exercise; no personal identifying data were provided. The numbers of recruits not present for training and those from a different BCT company were listed on the rosters and excluded from the analyses. Exposure categories were assigned to each group by their CS exposure concentration: $0.00-0.39 \mathrm{mg} / \mathrm{m}^{3}, 0.40-1.00 \mathrm{mg} / \mathrm{m}^{3}, 1.01-1.50 \mathrm{mg} / \mathrm{m}^{3}$, and greater than $1.50 \mathrm{mg} / \mathrm{m}^{3}$. Exposure categories were based on ACGIH TLV-C [skin] $\left(0.39 \mathrm{mg} / \mathrm{m}^{3}\right)$, one-half IDLH $(1.00$ $\left.\mathrm{mg} / \mathrm{m}^{3}\right)$ and three-fourths IDLH $\left(1.50 \mathrm{mg} / \mathrm{m}^{3}\right)[7,8]$.

The US Occupational Safety and Health Administration (OSHA) modified NIOSH Physical and Chemistry Analytical Method (P\&CAM) 304 was used to determine CS concentration for each exposure group [13, 14]. Personal sampling of individual recruits was not feasible; however previous studies showed a single static sample located eight meters from the entrance in the center of this particular chamber could characterize CS concentration during MCT and be used as a surrogate for individual CS exposures [9]. As with previous work, this study used a sampling train consisting of an OSHA Versatile Sampler (OVS) suspended $1.37 \mathrm{~m}$ above the floor (to represent the human breathing zone) connected to an air sampling pump using a $1.4 \mathrm{~m}$ section of Tygon tubing. The OVS contains both a filter and sorbent material, ideal for collecting both CS aerosol and vapors. The pump used was calibrated onsite to 1.5 liters per minute each day before sampling (and validated again after sampling). The pump was started when the first recruit of an exposure group entered the chamber and stopped when the last recruit of the exposure group exited. Total sampling time was annotated from the digital readout on the sampling pump and sample media was then capped, sealed in a plastic bag, and placed outside of the chamber in an ice filled cooler. New OVS media was then installed onto the pump and sampling continued until all exposure groups completed training.

In addition to static sampling, chamber operators were personally monitored using the same sampling train and flow rate as described above. The OVS media was clipped within six inches of the operator's nose and the pump was started when the operator entered the chamber. The pump was 
stopped only after the entire 200 recruit unit completed training and the operator departed the chamber. Total sampling time was annotated and samples were capped, sealed in individual bags, packaged on ice with daily area monitoring samples, and shipped within 24 hours to a certified laboratory for analysis.

Health surveillance data was captured using the same methodology described in previous work [10]. Clinically diagnosed ARI case counts for each company completing the MCT were captured through existing weekly Fort Jackson Acute Respiratory Disease Surveillance (ARDS) systems [15]. This surveillance program queried the Composite Healthcare Computer System (CHCS) for clinically diagnosed ARI encounters within the Fort Jackson recruit population matching one or more of the following International Classification of Diseases Version 9 (ICD-9) codes: 079.99 Viral infection, not otherwise specified (NOS); 382.9 Otitis media NOS; 460 Nasopharyngitis, acute; 461.9 Acute sinusitis; 465.8 Acute upper respiratory infections of other multiple sites; 465.9 Acute upper respiratory infections of unspecified site; 466.0 Bronchitis, acute; 486 Pneumonia, organism NOS; 487.0 Influenza with pneumonia; 487.1 Influenza with respiratory manifestation, not elsewhere classified (NEC); 487.8 Influenza with manifestation NEC; 490 Bronchitis NOS; 784.1 Pain, Throat; and 786.2 Cough [10]. Preventive medicine personnel used these data combined with military training unit specific attendance rosters to determine case and exposure group status for recruits diagnosed with ARI during their company's surveillance period. For the purposes of the present study, the surveillance period started seven days before each unit's chamber training and ended seven days after completion. Recruits who completed training with their unit and were diagnosed with ARI in the surveillance period were designated as cases. Incidence rules provided to preventive medicine personnel dictated that the first diagnosis of ARI during a surveillance period was used as the date of encounter; however if an afebrile case progressed to a febrile case later in the surveillance period, date of febrile diagnosis took precedence. A febrile case was defined as diagnosis with one or more of the aforementioned ICD-9 codes and an oral temperature greater than $38.1^{\circ} \mathrm{C}$. Investigators were provided ARI count data in a format that summarized the number of incident cases by training unit, exposure group, date of medical encounter, and febrile/afebrile status. Personal identifying data were not provided to investigators (Table 1). Investigators used the date of encounter to determine if a case occurred in the week preceding the chamber (pre-chamber ARI) or in the sevendays following the chamber (post-chamber ARI). Pre- chamber ARI cases were treated as non-susceptible for postchamber ARI and were excluded from post-chamber risk calculations.

CS concentrations were matched to corresponding military unit, exposure group data, and ARI incidence and entered into SPSS Statistics for Windows (Version 19, IBM Corp., 2010) for data management. Open Source Epidemiologic Statistics for Public Health (Version 3.01, www.openepi.com., 2013) was used to calculate chi-squared values, Breslow-Day tests for interaction, stratified risks, attributable risks, as well as unadjusted and Mantel-Haenszel adjusted risk ratios (RR) with corresponding $95 \%$ confidence intervals $(95 \% \mathrm{CI})$. Statistical Package for the Social Sciences (SPSS) was used to calculate means, standard deviations, Shapiro-Wilk tests, and 95\% confidence intervals for CS concentrations. SPSS was also used to conduct Poisson regression analyses to explore the relationship between CS exposure concentrations and ARI outcomes.

\section{RESULTS}

\section{Exposure Assessment}

Table 2 depicts the CS exposure data for trainees and chamber operators in this study. Recruit CS exposure concentrations were observed from $0.26-2.78 \mathrm{mg} / \mathrm{m}^{3}$ $\left(\bar{x}=1.04 \mathrm{mg} / \mathrm{m}^{3}\right)$ over 106 unique MCT events with exposure durations ranging from $5.0-25.0$ minutes $(\bar{x}=11.67 \mathrm{~min})$. Personal monitoring of six chamber operators over 28 sampling episodes demonstrated CS exposure concentrations ranging from $0.05-2.22 \mathrm{mg} / \mathrm{m}^{3}\left(\bar{x}=1.05 \mathrm{mg} / \mathrm{m}^{3}\right)$ with exposure durations from $20.0-162.0$ minutes $(\bar{x}=62.73$ $\mathrm{min}$ ). Trainee eight-hour time weighted averages (TWA) ranged from $0.004-0.11 \mathrm{mg} / \mathrm{m}^{3}\left(\bar{x}=0.02 \mathrm{mg} / \mathrm{m}^{3}\right)$ and chamber operator TWA ranged from $0.02-0.35 \mathrm{mg} / \mathrm{m}^{3}$ $\left(\bar{x}=0.12 \mathrm{mg} / \mathrm{m}^{3}\right)$. Nearly $90 \%$ of area samples $(\mathrm{n}=94)$ and personal monitoring samples $(n=24)$ exceeded the ACGIH TLV-C [skin] $\left(0.39 \mathrm{mg} / \mathrm{m}^{3}\right)$; five of these (four area samples and 1 personal monitoring sample) exceeded the IDLH (2.00 $\left.\mathrm{mg} / \mathrm{m}^{3}\right)$. Neither trainees nor operators exceeded the 8-hour OSHA PEL $\left(0.40 \mathrm{mg} / \mathrm{m}^{3}\right)$ [16].

\section{Outcome Assessment}

Of the 5298 recruits who participated in MCT during the surveillance period, the majority $(55 \%)$ completed the MCT during week two of $\mathrm{BCT}$ and $96 \%$ completed the training during the first three weeks of BCT (Table 3). Trainees lived in one of three building types: 1) Starship barracks (SS) Housing units with multiple 60-person rooms; 2) Relocatable barracks (RL) - Temporary modular structures capable of housing up to 50-people per room; and 3) Star base barracks

Table 1. Example of de-identified ARI health outcome data provided to investigators.

\begin{tabular}{|c|c|c|c|c|c|}
\hline \multirow{2}{*}{ Company (UIC) } & Chamber Date & Case \# & Encounter Date & Febrile/Afebrile & Exposure Group \\
\hline \hline \multirow{2}{*}{ WHG01 } & \multirow{3}{*}{13 Mar 2013 } & 1 & 10 Mar 2013 & A-Feb & Feb \\
\cline { 3 - 6 } & & 2 & 15 Mar 2013 & A-Feb & 2 \\
\cline { 3 - 6 } & \multirow{2}{*}{ WHG02 } & 3 & 13 Mar2013 & A-Feb & A-Feb \\
\cline { 3 - 6 } & & 1 & 12 Mar 2013 & 19 Mar 2013 & 1 \\
\hline
\end{tabular}


(SB) - Newer housing units with multiple 60-person rooms. Nearly half of the recruits $(45 \%)$ lived in relocatable barracks.

Table 2. CS exposure concentrations for trainees and chamber operators.

\begin{tabular}{|c|c|c|}
\hline \multicolumn{3}{|c|}{$\mathbf{N}(\%)$} \\
\hline Overall & $\begin{array}{c}\text { Trainees } \\
\mathbf{5 2 9 8}(\mathbf{1 0 0})\end{array}$ & $\begin{array}{c}\text { Chamber Operators } \\
\mathbf{2 8}(\mathbf{1 0 0})\end{array}$ \\
\hline \hline CS Concentration $\left[\mathrm{mg} / \mathbf{m}^{3}\right]$ \\
\hline$[\mathbf{0 . 0 0}-\mathbf{0 . 3 9}]$ & $600(11.33)$ & $4(14.29)$ \\
\hline$[\mathbf{0 . 4 0}-\mathbf{1 . 0 0}]$ & $2578(48.66)$ & $9(32.14)$ \\
\hline$[\mathbf{1 . 0 1}-\mathbf{1 . 5 0}]$ & $1063(20.06)$ & $9(32.14)$ \\
\hline$[\mathbf{1 . 5 1}-\mathbf{2 . 0}] \mathbf{0}$ & $702(13.25)$ & $5(17.86)$ \\
\hline$[>\mathbf{2 . 0}]$ & $355(6.70)$ & $1(3.57)$ \\
\hline
\end{tabular}

Exceeded ACGIH TLV-C $\left[0.39 \mathrm{mg} / \mathrm{m}^{3}\right]$.

Exceeded NIOSH IDLH $\left[2.0 \mathrm{mg} / \mathrm{m}^{3}\right]$.

There were 155 clinically diagnosed cases of ARI (4 febrile and 151 afebrile) observed in the study population. Approximately one-third $(n=56)$ of these cases occurred in the seven days prior to CS exposure (pre-chamber) and the
Table 3. ARI incident cases by week the CS chamber occurred and building type.

\begin{tabular}{|c|c|c|c|}
\hline & \multicolumn{3}{|c|}{ N (\%) } \\
\hline Overall & $\begin{array}{c}\text { Pre-Chamber } \\
\text { ARI 56 (1.06) }\end{array}$ & $\begin{array}{c}\text { Post-Chamber } \\
\text { ARI 99 (1.87) }\end{array}$ & $\begin{array}{c}\text { Total } \\
\text { Population 5298 }\end{array}$ \\
\hline \hline Chamber Week \\
\hline $\mathbf{1}$ & $9(0.78)$ & $17(1.46)$ & 1166 \\
\hline $\mathbf{2}$ & $37(1.26)$ & $64(2.19)$ & 2929 \\
\hline $\mathbf{3}$ & $9(0.89)$ & $17(1.68)$ & 1009 \\
\hline $\mathbf{8}$ & $1(0.01)$ & $1(0.01)$ & 194 \\
\hline Building Type & $21(1.21)$ & $39(2.25)$ & 1737 \\
\hline SS & $20(0.84)$ & $39(1.64)$ & 2378 \\
\hline RL & $15(1.27)$ & $21(1.78)$ & 1183 \\
\hline SB & & & \\
\hline
\end{tabular}

remaining two-thirds $(\mathrm{n}=99)$ occurred during the seven days following CS exposure (post-chamber). Table 4 shows that the overall ARI risk was significantly higher in the seven days following exposure to CS compared to the seven-day period before the exposure $(\mathrm{RR}=1.79,95 \% \mathrm{CI}=1.29,2.47)$.

Table 4. ARI rates and attributable risks (per 100 person-weeks) by chamber week, building type, and CS concentration.

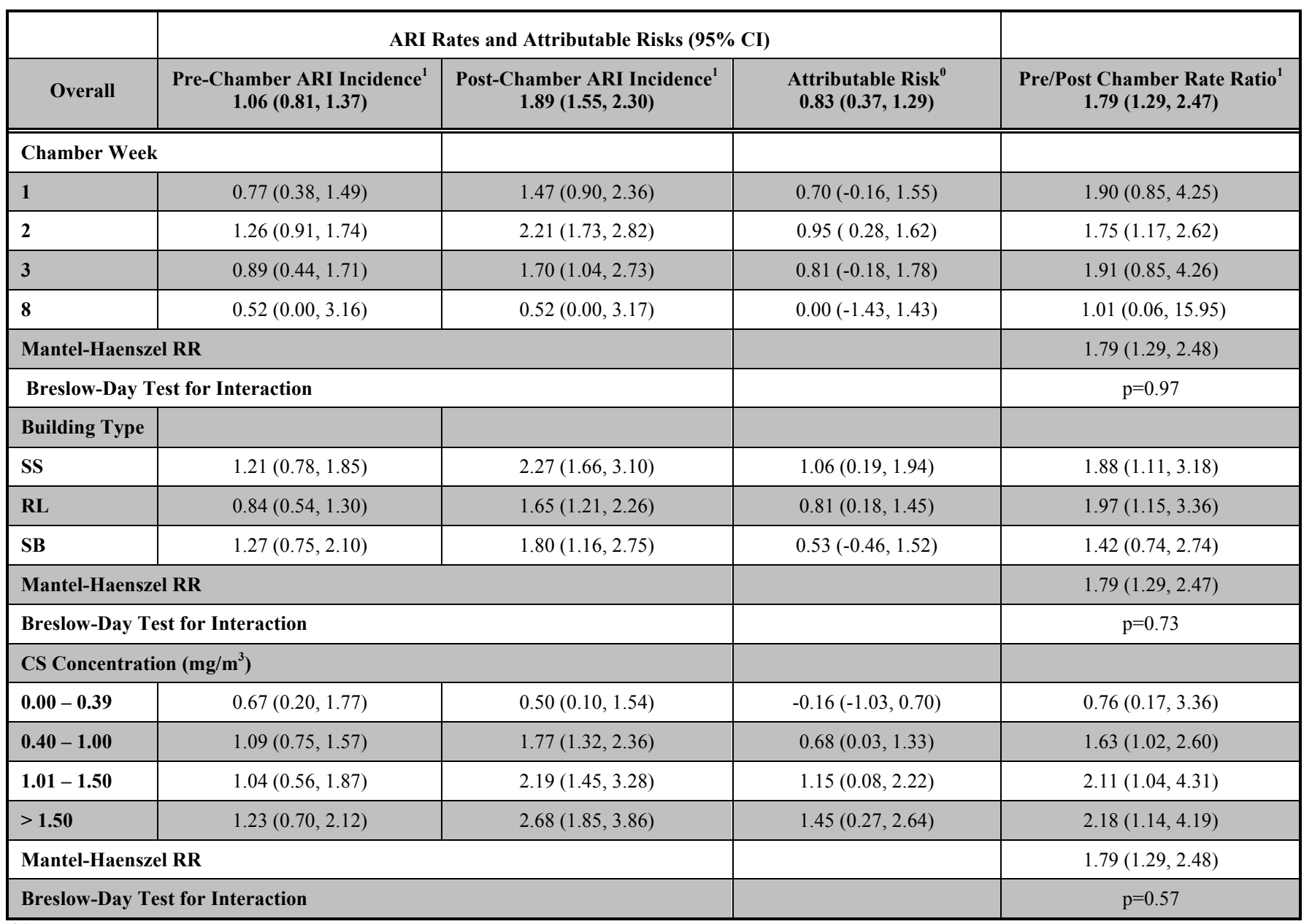

( ${ }^{1} 95 \%$ confidence limits testing the exclusion of 0 or 1 , as indicated). 
Increased ARI risk was observed independent of the week of CS exposure or barracks building type. Stratum specific Mantel-Haenszel adjusted rate ratios did not suggest confounding and the Breslow-Day test for interaction did not suggest interaction by either variable. A chi-squared test for homogeneity revealed no significant difference between prechamber ARI incidence rates across chamber weeks $(\mathrm{p}=0.37)$, building types $(\mathrm{p}=0.38)$, CS concentration categories $(\mathrm{p}=0.75)$; or post-chamber ARI incidence rates with respect to chamber week $(\mathrm{p}=0.18)$ or building type $(\mathrm{p}=0.35)$. However, a chi-squared test for independence suggested that post-chamber ARI rates $(p=0.02)$ were dependent upon CS exposure concentration.

A Poisson regression analysis showed a significant difference between pre and post-chamber ARI rates across all concentration categories $(\mathrm{p}=0.049)$ and significantly elevated pre/post-chamber ARI rate ratios at all concentration categories higher than the ACGIH TLV-C [skin]. These elevated rate ratios however, were not statistically different from each other $(\mathrm{p}=0.58)$ (Fig. 1).

\section{DISCUSSION}

Comparing the results of this study with those conducted before ALARACT 051/2013 implementation shows that the change in Army MCT procedures resulted in an approximately 10 -fold decrease in the CS concentration experienced by trainees and chamber operators. It suggests implementation also resulted in a lower risk of ARI diagnosis in the seven-day period following exposure to CS when compared to the seven-day period before CS exposure and further demonstrates post-exposure ARI incidence is dependent upon CS exposure concentration.

Fig. (2) compares CS concentrations before and after implementation of ALARACT 051/2013 to illustrate the significant reduction $(\mathrm{p}<0.001)$ in mean CS concentrations for both groups and how these align with established US exposure guidelines. This decrease resulted in fewer recruits being exposed to $\mathrm{CS}$ concentrations exceeding $\mathrm{NIOSH}$ IDLH (98\% reduced to 7\%), ACGIH TLV-C [skin] (100\% reduced to $89 \%)$, and the OSHA PEL (12\% reduced to $0 \%)$ compared to recruit exposures before implementation [9].

The reduction in CS exposure concentrations is likely due to the decrease in CS used to conduct the MCT. Prior to the recent procedural changes discussed here, MCT required the dispersal of one- $650 \mathrm{mg}$ CS capsule for every $30 \mathrm{~m}^{3}$ of chamber size to establish an initial CS concentration, followed by one additional capsule for every 10 recruits exiting the chamber to maintain the CS concentration $[3,4]$. ALARACT 051/2013 specified a new CS dosage formula (\# of capsules $=$ [volume $\left.\left.\left(\mathrm{m}^{3}\right) * 0.0107\right]\right)$ that required dispersal of only 0.32 CS capsules for every $30 \mathrm{~m}^{3}$ to establish the initial CS concentration, followed by one additional capsule for every 50 recruits exiting the chamber to maintain the CS concentration [11]. This resulted in nearly two-thirds reduction in the mass of CS used to charge the chamber and an $80 \%$ reduction in the mass of CS used to maintain the CS concentration. This reduced CS mass likely resulted in the observed lower airborne CS concentrations experienced by recruits and chamber operators.

Despite chamber operators strictly adhering to the CS dosage instruction of the ALARACT, CS concentrations were not normally distributed $(\mathrm{p}<0.001)$ and ranged from $0.26-2.78 \mathrm{mg} / \mathrm{m}^{3}$ for trainees and $0.05-2.22 \mathrm{mg} / \mathrm{m}^{3}$ for chamber operators. This distribution is likely due to uncontrolled factors such as the number of times the doors of the chamber were opened and the number of times the chamber operator exited and returned to the chamber. If a recruit's mask was not properly sealed, they were asked to open the door and exit the chamber. Chamber operators often followed the recruit to ensure the mask was properly sealed before allowing chamber re-entry. This recurring scenario may have enabled aerosolized CS to escape the chamber and

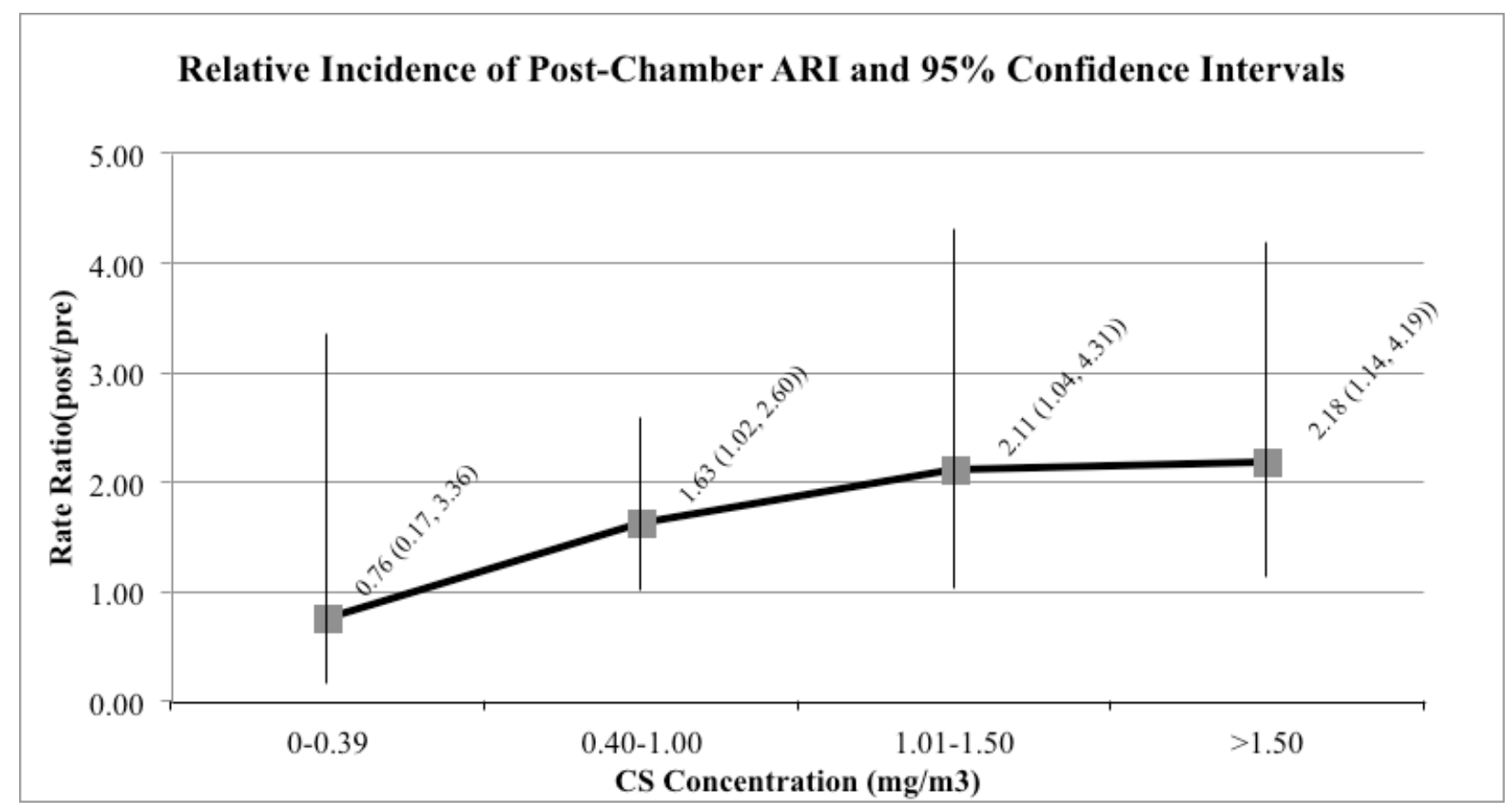

Fig. (1). Relative incidence of post-chamber ARI by CS concentration and $95 \%$ confidence intervals. 


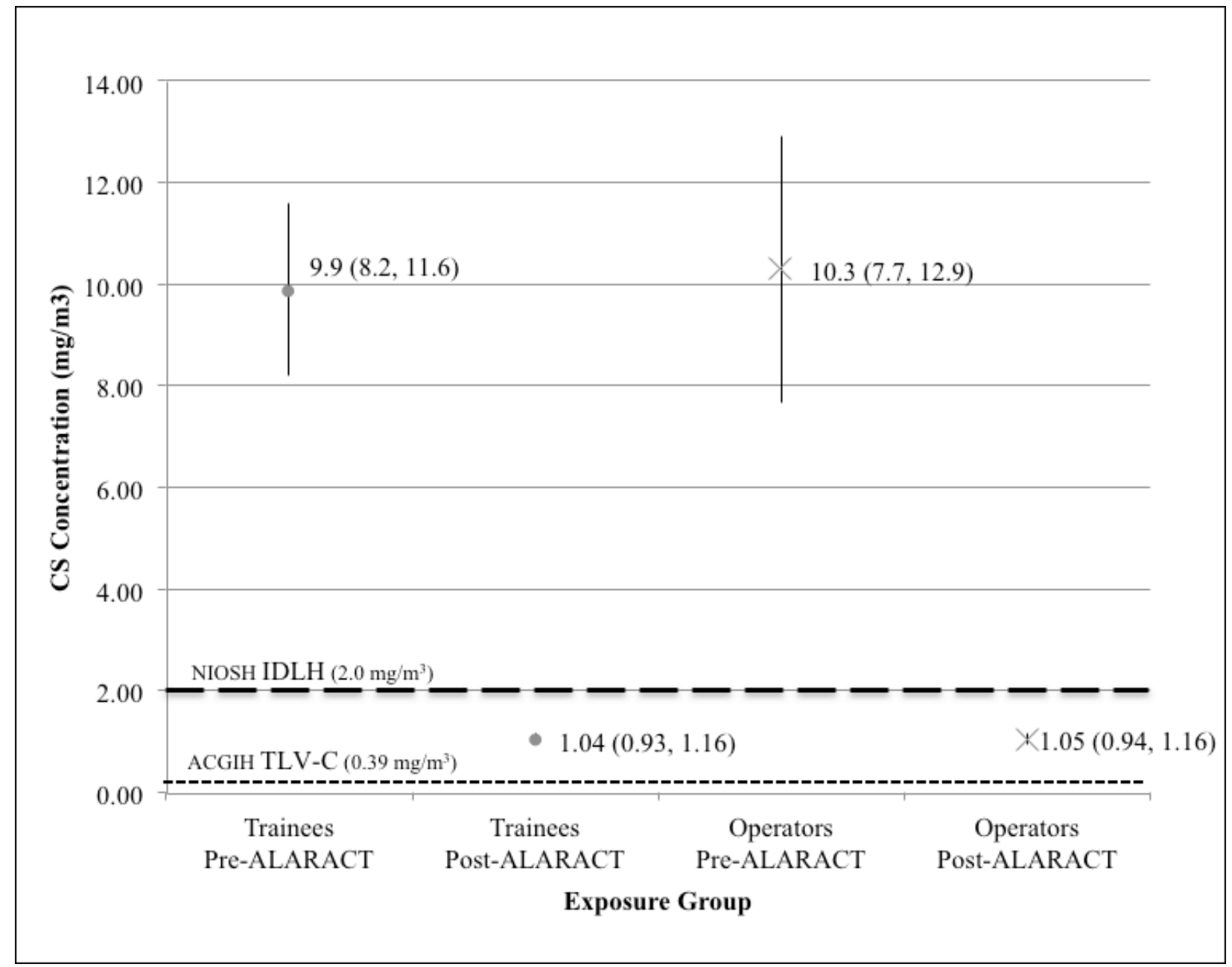

Fig. (2). CS Concentration by Exposure Group, Pre and Post ALARACT. • Represents trainee mean exposure concentration; X represents operator mean exposure concentration; vertical bars represent $95 \%$ confidence interval.

dilution ventilation to enter thus decreasing the CS concentration experienced by participants. During situations such as this, the personal sampling pump on the respective chamber operator was not paused, which could have also resulted in a decreased CS concentration measurement for chamber operators.

In addition to the decrease in $\mathrm{CS}$ concentration, recruit time required in the chamber without their protective mask was also reduced with the ALARACT. In accordance with US Army safety regulations, recruits would no longer be exposed to CS without respiratory protection beyond 15 seconds [17]. Chamber operators strictly enforced this standard and unprotected exposures lasting longer than 15 seconds were not observed. In fact, nearly half of the population only removed masks for durations of less than five seconds. Even in this scenario however, recruits were still exposed via the dermal pathway for the duration of the chamber exercise.

The aforementioned changes may have contributed to the reduction in risk of post-chamber ARI diagnoses observed in this study compared with previous work. After implementation of the ALARACT, recruits had a 1.79 times greater risk of ARI diagnosis within the seven days following exposure to CS compared to the seven days before
CS exposure. Similar research conducted before the ALARACT demonstrated a 2.44 times greater risk for ARI diagnosis using the same pre and post-chamber surveillance periods [10]. In both pre and post ALARACT studies, week of chamber training and the barracks building type did not play a significant role in ARI outcomes during the surveillance period. This contradicts previous ARI studies that demonstrated that both building type and week of training had an impact on ARI outcomes [18]. Previous studies however, were conducted before mandatory adenovirus type 4 and 7 vaccine was reintroduced in this population [19]. This vaccine has been shown to significantly decrease febrile ARI incidence among the BCT recruit population across US Department of Defense initial military training sites [20]. Observed ARI disease patterns may have changed due to the impact of these vaccines; as such it is difficult to make comparisons of studies conducted before and after its implementation.

The lack of an observed relationship between prechamber ARI and CS exposure concentrations $(p=0.75)$ coupled with pre-chamber ARI rates that were not significantly different nor elevated at any observed CS concentration levels suggested pre-chamber ARI was independent of CS exposure. Incidence of ARI after CS exposure however, was temporally linked to the exposure 
and was associated with $\mathrm{CS}$ exposure concentrations $(p=0.02)$. Risk of being diagnosed with ARI after exposure to CS was significantly elevated in all concentrations higher than the ACGIH-TLV-C [skin] $\left(0.39 \mathrm{mg} / \mathrm{m}^{3}\right)$. There was a statistical difference between the post-chamber ARI rate in the highest CS concentration category $\left(>1.5 \mathrm{mg} / \mathrm{m}^{3}\right)$ compared to the lowest $\left(0.00-0.39 \mathrm{mg} / \mathrm{m}^{3}\right) \quad(\mathrm{p}=0.02)$. Pre/post-chamber rate ratios were also significantly elevated in all concentrations above the ACGIH-TLV-C [skin] but were not statistically different than each other (Table 4).

The elevated post-chamber ARI rates and pre/postchamber rate ratios at concentrations above $0.39 \mathrm{mg} / \mathrm{m}^{3}$ are suggestive of a protective effect of the ACGIH-TLV-C [skin] compared to CS exposure concentrations exceeding this value. This is further supported by an observed decrease in both the post-chamber ARI rate $(0.50,95 \% \mathrm{CI}=0.10,1.54)$ and pre/post-chamber rate ratio $(\mathrm{RR}=0.76,95 \% \mathrm{CI}=0.17$, 3.36 ) in this CS concentration range when compared to other groups. However, this reduction in risk was based upon only $11 \%(\mathrm{n}=600)$ of the population and was not statistically significant. These results are consistent with previous research suggesting CS exposures below IDLH $\left(2.00 \mathrm{mg} / \mathrm{m}^{3}\right)$ decreased ARI risk when compared to higher CS exposure concentrations [10]. However, the previous study had only $2 \%(n=134)$ exposed to $\mathrm{CS}$ at this concentration range making it difficult to draw conclusions regarding ARI risks at concentration gradients below IDLH. In the current study, implementation of ALARACT 051/2013 resulted in exposure of $93 \%$ ( $n=4943)$ of the study population to CS concentrations less than $2.00 \mathrm{mg} / \mathrm{m}^{3}$. This increase in sample size enabled more detailed analysis at this level and suggested a gradual reduction in elevated ARI risk as CS concentrations levels approach ACGIH-TLV-C [skin] with a potential threshold for doubling of ARI risk in the range of $0.39-1.00 \mathrm{mg} / \mathrm{m}^{3}$. These results are also consistent with previous research suggesting a threshold effect in the range of $0.00-2.00 \mathrm{mg} / \mathrm{m}^{3}[10]$.

Table 5 illustrates the differences between ARI rates before and after implementation of ALARACT 051/2013. The overall, pre-chamber, and post-chamber ARI rates increased after implementation of the ALARACT; however only differences in pre-chamber ARI rates reached statistical significance $(\mathrm{p}=0.04)$. These results were expected since seasonality has been shown to be a predictor of ARI incidence in this population $[18,21]$. The pre-ALARACT study was conducted from August - September 2012 before the start of the cold and flu season and demonstrated a febrile ARI incidence rate of 0.030 cases $/ 100$ person-weeks and an afebrile ARI incidence rate of 1.20 cases/100 personweeks [10]. During this same period, the febrile ARI incidence rate for all trainees attending BCT at Fort Jackson, $\mathrm{SC}$ during this period was 0.087 cases/100-person-weeks [22]. This may have resulted in lower baseline ARI incidence than the present study, which was conducted near the end of the cold and flu season with a higher febrile ARI incidence rate of 0.038 cases $/ 100$ person-weeks and an afebrile ARI incidence rate of 1.40 cases/100 person-weeks in this study population. The febrile ARI incidence rate for all recruits attending training at Fort Jackson during this same time period was 0.091 cases/100 person-weeks [23]. Despite the significantly higher baseline ARI rate observed in this study, the adjusted pre/post-chamber ARI rate ratio decreased after implementation of ALARACT 051/2013 resulting in $26.85 \%(95 \% \mathrm{CI}=-0.17,0.54)$ effectiveness for the intervention; however neither the difference in the rate ratios nor the effectiveness of the intervention achieved statistical significance $(\mathrm{p}=0.19)$. It still suggestive however, that this intervention may have decreased ARI risks associated with exposure to $\mathrm{CS}$ in this US Army BCT cohort.

Table 5. Comparison of Pre and post ALARACT ARI rates (per 100 person-weeks) and their $95 \%$ confidence intervals.

\begin{tabular}{|c|c|c|c|}
\hline & Pre-ALARACT & Post-ALARACT & P Value \\
\hline \hline Overall ARI Rate & $2.39(2.05,2.78)$ & $2.93(2.49,3.41)$ & 0.07 \\
\hline Pre-Chamber ARI Rate & $0.70(0.52,0.93)$ & $1.06(0.81,1.37)$ & 0.04 \\
\hline Post-Chamber ARI Rate & $1.71(1.42,2.05)$ & $1.89(1.54,2.29)$ & 0.46 \\
\hline MH Adjusted ARI Rate Ratio & $2.44(1.74,3.43)$ & $1.79(1.29,2.48)$ & 0.19 \\
\hline
\end{tabular}

This study demonstrated a lower ARI incidence rate in $\mathrm{BCT}$ than those presented historically, which is consistent with the study conducted before implementation of ALARACT 051/2013. This lower rate was expected due to the implementation of the Adenovirus 4 and 7 vaccines in 2011 that has been shown to significantly decrease ARI burden in this population $[20,24,25]$. There were only four febrile ARI cases in the study population, one case presented before exposure to CS and the other three were diagnosed post-chamber. All post-chamber cases were exposed to CS in concentrations higher than the ACGIH TLV-C [skin]. This may suggest increased concentrations of CS increase the risk of febrile ARI; however this is difficult to determine based upon such sparse data. It also may suggest the large proportion $(97 \%)$ of afebrile post-chamber cases in this population are respiratory irritation caused by the effects of $\mathrm{CS}$ rather than infection. Post-chamber ARI diagnoses due to irritation would be expected within 24 hours of MCT completion since symptoms associated with CS exposure typically resolve only minutes after cessation of exposure. However, only $5.05 \%$ of post-chamber ARI presented within 24 hours of MCT completion. In fact, only $17.17 \%$ of postchamber ARI diagnoses occurred within the two days following MCT. This may suggest that CS induced stress on the respiratory tract increases susceptibility to ARI causing pathogens, resulting in a higher post-chamber ARI incidence rate and more cases later in the follow-up period.

Fig. (3) combines the data from the pre-ALARACT study with the results obtained in this research to cover a range of CS concentrations from $0.26-55.24 \mathrm{mg} / \mathrm{m}^{3}$ [10]. A Poisson regression analysis showed a significant difference between pre and post-chamber ARI rates at all concentration levels $(\mathrm{p}<0.001)$; the risk of being diagnosed with ARI before the chamber compared to the risk of being diagnosed after the chamber was significantly elevated in all concentration categories above the ACGIH-TLV-C [skin] with the exception of $1.50-2.00 \mathrm{mg} / \mathrm{m}^{3}$. The lack of statistical significance in this concentration category is likely due to sparseness of data as only $7 \%(n=836)$ of the combined population was exposed at these concentrations. The 


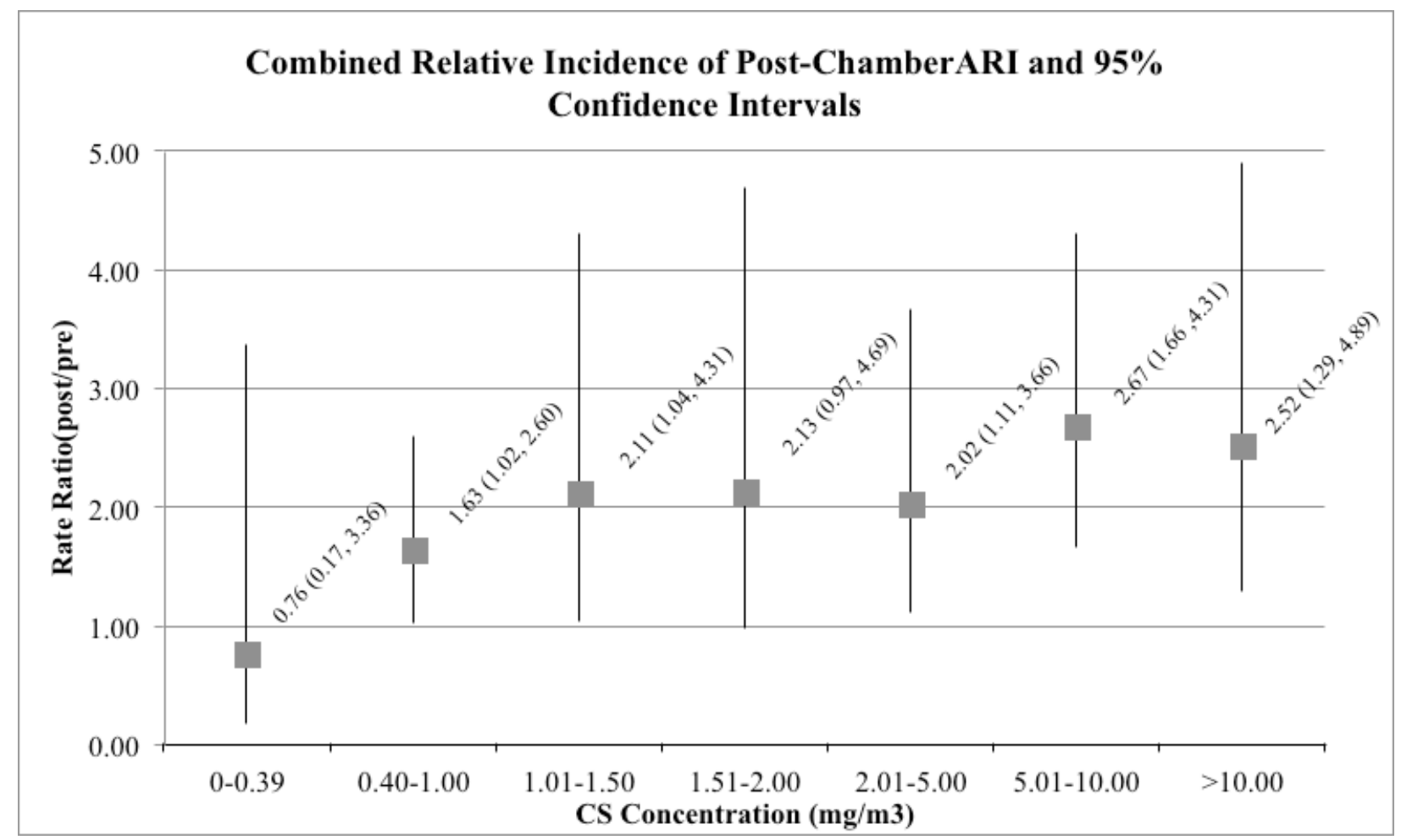

Fig. (3). Combined relative incidence of post-chamber ARI by CS concentration and $95 \%$ confidence intervals.

combined data reveal that post-chamber incidence was dependent upon CS exposure concentrations $(\mathrm{p}=0.048)$ and further supports the suggestion of lower ARI risk at CS concentrations below the ACGIH TLV-C [skin] followed by an increase in risk to a threshold concentration between 0.40 and $1.00 \mathrm{mg} / \mathrm{m}^{3}$ where the risk approximately doubles and remains constant. One must be cautious in interpretation of these results however as the analysis failed to detect a difference between rate ratios at the $95 \%$ confidence level $(\mathrm{p}=0.65)$.

There are several limitations associated with this observational study. Investigators were not permitted to influence the training, CS concentrations, CS exposure times, or perform any actions that had the potential to disrupt training which led to limitations in the exposure assessment portion of this study. For example, personal monitoring had the potential to disrupt training, therefore CS exposure concentrations were based upon area sampling rather than personal monitoring. This methodology assumed that CS was evenly dispersed throughout the chamber and that all recruits within an exposure group were exposed to the same level of CS based upon one static sample. While this is an acceptable method to estimate exposures to a group of people, it does not show the variability that would be expected within a given exposure group based upon location in the chamber and number of times the door was opened [14]. Furthermore, exposure time and time with mask removed often varied from recruit to recruit within exposure groups and this variability was not captured. The small amount of data in the lowest concentration category makes it difficult to determine if there is a truly a protective effect afforded by the ACGIH TLV$\mathrm{C}$ [skin]. Future studies should consider lower CS exposure concentrations to better explore health outcomes related to CS exposures occurring below the ACGIH TLV-C [skin].
Another limitation lies in the inability of this study to differentiate between acute respiratory irritation and acute respiratory infection. Health outcomes were based solely upon acute respiratory related ICD- 9 codes that appeared in electronic medical records, making it difficult to determine if symptoms were due to $\mathrm{CS}$ induced irritation of the respiratory tract or if they were the beginning of a respiratory infection. Furthermore, the brief surveillance period used here may not have been sufficient to identify afebrile cases that later progressed to febrile cases. Given this limitation, the number of febrile incident cases observed was small with febrile ARI rates lower than those observed by Army Public Health Command across the entire Fort Jackson trainee population during the study period (0.030 vs 0.087 cases/100-person weeks) [23]. Previous studies however show that febrile ARI rates tend to peak during weeks four through six of Army BCT, and this study did not capture these data since it focused on health outcomes associated with mask confidence chamber training generally completed during the first three weeks of BCT $[18,19]$. Without surveillance data for each company for the entire training cycle, it is difficult to determine whether the trends observed here are due to CS exposure or are part of an underlying disease trend that peaks later in the BCT cycle.

\section{CONCLUSION}

This study demonstrated that the implementation of the controls outlined in ALARACT 051/2013 resulted in a ten-fold reduction in the $\mathrm{CS}$ exposure concentrations experienced by US Army recruits attending BCT at Fort Jackson, S.C. from 13 March to 30 April 2013. It also suggested this intervention may have resulted in a decreased ARI risk in the seven days following mandatory MCT compared to the seven days before exposure when compared to studies conducted before ALARACT implementation. Results from the present study 
further support findings from previous research that suggest the risk of ARI diagnosis after CS exposure is positively associated with CS concentration experienced by recruits.

Despite the success of this intervention in lowering CS concentrations below the IDLH, mean recruit CS concentrations still exceed the ACGIH TLV-C [skin]. As such, efforts should be made to further decrease CS concentrations since this research suggested a decreased ARI risk for CS concentrations below this level. Demonstrating the effectiveness of the military protective mask can be achieved at CS concentrations as low as the odor threshold $\left(0.004 \mathrm{mg} / \mathrm{m}^{3}\right)$ [2]. Furthermore, since CS bears a skin notation, efforts should be made to protect exposed skin through the use of chemical protective garments during this training [7].

Future research is needed to better characterize ARI health outcomes reported here by differentiating between CS induced irritation or infection, and to explore these outcomes at CS concentrations below the ACGIH TLV-C [skin]. Research is also needed to determine if this intervention resulted in decreased hospital burden and lost training time in the BCT population.

\section{CONFLICT OF INTEREST}

The authors confirm that this article content has no conflict of interest.

\section{ACKNOWLEDGEMENTS}

This work was sponsored by the US Army Medical Command, Office of the Surgeon General (MEDCOM/ OTSG), Falls Church, Virginia. Laboratory and technical support were provided by the Army Institute of Public Health (AIPH), Aberdeen Proving Ground, Maryland.

\section{DISCLAIMER}

The views expressed in this article are those of the authors and do not reflect the official policies or positions of the Uniformed Services University of the Health Sciences, Department of the Army, Department of Defense, or the US Government.

\section{REFERENCES}

[1] Hout J, Kluchinsky T, Peter T, White DW. Identification of Compounds Formed during the Low Temperature Heat Dispersal of Encapsulated o-Chlorobenzylidene Malononitrile (CS Riot Control Agent). J Occup Environ Health 2010; 7(6): 352-7.

[2] Hout J, Hout JJ, Kluchinsky T, LaPuma PT, White DW . Evaluation of CS (o-chlorobenzylidene malononitrile) Concentrations During U.S. Army Mask Confidence Training. J Environ Health 2011; 74(3): 18-21.

[3] Department of the Army, Training Circular (TC) 3-8, Chemical Training. Washington, D.C. 1994; pp. 3.1-3.6.

[4] Department of the Army, Chemical, Biological, Radiological, and Nuclear (CBRN) Defense Mask Confidence Training Procedures: Training Support Package 805-B-2040, 2008.
[5] Department of the Army, Field Manual (FM) 3-11.11, Flame, Riot Control Agent, and Herbicide Operations. Department of the Army: Washington D.C. 2003; pp. 6-2.

[6] Department of the Army, Department of the Army Pamphlet (DA PAM) 350-38. Washington, D.C. Standards in Training Commission 2012; p. 15.

[7] ACGIH, TLVs and BEIs 2010, Cincinnati, OH: American Conference of Governamental Industrial Hygienists.

[8] NIOSH, Documentation for Immediately Dangerous To Life or Health Concentrations (IDLHs). Cincinnati, OH 1994.

[9] Hout J. O-Chlorobenzylidene Malononitrile (CS Riot Control Agent) Exposures in a U.S. Army Basic Combat Training Cohort. J Environ Health, October 2014; (in press).

[10] Hout, J. O-Chlorobenzylidene Malononitrile (CS Riot Control Agent) Associated Acute Respiratory Illnesses in a U.S. Army Basic Combat Training Cohort. Mil Med 2014; 179(7): 793-8.

[11] U.S. Army Safety Office, Alaract 051/2013: safety alert on mask confidence training (MCT) procedures using o-chlorobenzylidene malononitrile (CS) Capsules 2013; pp. 1-2.

[12] Office of Research, U.S.U., Notice of Project Approval: T08768-01 O-Chlorobenzylidene Malononitrile Exposures and Acute Respiratory Rates in an Army Basic Training Population, Research, Editor: Bethesda, MD 2012.

[13] NIOSH, o-Chlorobenzylidene Malononitrile (OCBM), Physical and Chemical Analytic Method (P\&CAM) 304, 1979.

[14] OSHA. Chemical Exposure Health Data. [Cited: 2013, 21 Feb]; Available from: http://www.osha.gov/opengov/healthsamples.html.

[15] Department of the Army, Army Acute Respiratory Disease Surveillance Program, Office of the Surgeon General, Editor 2010: Falls Church, VA. p. 4.

[16] OSHA, Occupational Health Guideline for o-Chlorobenzylidene Malononitrile, 1976, U.S. Department of Health and Human Services, Public Health Service, Centers for Disease Control, National Institute for Occupational Safety and Health: Cincinnati, $\mathrm{OH}$.

[17] Department of the Army, Department of the Army Pamphet (DA PAM) 385-63, Range Safety, Department of the Army: Washington, DC. 2012; pp. 160-1.

[18] White D, McKeown R, Hout J, Hebert J. Association Between Barracks Type and Acute Respiratory Infection in a Gender Integrated Army Basic Combat Training Population. Mil Med 2011; 176(2:909): 909-14.

[19] Kolavic-Gray SA, Binn LN, Sanchez JL. Large epidemic of adenovirus type 4 infection among military trainees: epidemiological, clinical, and laboratory studies. Clin Infect Dis 2002; 35(7): 808-18.

[20] Hoke CH, Snyder C. Initial assessment of impact of adenovirus type 4 and type 7 vaccine on febrile respiratory illness and virus transmission in military basic trainees, March 2012. Med Surveill Mon Rep 2012; 19(3): 2-5.

[21] Top FH. Control of adenovirus acute respiratory disease in U.S. Army trainees. Yale J Biol Med 1975; 48(3): 185-95.

[22] United States Army Public Health Command. Acute Respiratory Disease Report. USAPHC: August - September 2012, 2012.

[23] United States Army Public Health Command, Acute Respiratory Disease Surveillance Weekly Report: 9 March - 4 May 2013, 2013.

[24] Armed Forces Health Surveillance Center, Incidence of acute respiratory illnesses among enlisted service members during their first year of military service: did the 2011 resumption of adenovirus vaccination of basic trainees have an effect? Med Surveil Mon Rep 2013; 20(5): 14-8

[25] Armed Forces Health Surveillance Center, Update: pneumoniainfluenza and severe acute respiratory illnesses, active component, U.S. Armed Forces, July 2000-June 2012. Med Surveill Mon Rep 2012; 19(9): 11-5. [Editorial Comment 13-5]. 